\title{
Juvenile Myasthenia Gravis: Recommendations for Diagnostic Approaches and Treatment
}

\author{
${ }^{1}$ Department of Neuropediatrics, Developmental Neurology and \\ Social Pediatrics, University of Essen, Essen, Germany \\ Neuropediatrics 2014;45:75-83.
}

Adela Della Marina $^{1} \quad$ Heike Trippe $^{1} \quad$ Soeren Lutz $^{1} \quad$ Ulrike Schara $^{1}$

\begin{abstract}
Address for correspondence Dr. Adela Della Marina, MD, Department of Neuropediatrics, Developmental Neurology and Social Pediatrics, University of Essen, Hufelandstrasse 55, 45122 Essen, Germany (e-mail: adela.dellamarina@uk-essen.de).
\end{abstract}

\begin{abstract}
Keywords

- juvenile myasthenia gravis

- clinical symptoms

- differential diagnosis

- diagnostic workup

- therapy

Juvenile myasthenia gravis (JMG) is an autoimmune disorder of neuromuscular transmission caused by production of antibodies against components of the postsynaptic membrane of the neuromuscular junction. Ethnicity has influence on incidence, clinical presentation, and the course of the disease. The patients present with a wide range of symptoms-from isolated intermittent ocular symptoms to general muscle weakness with or without respiratory insufficiency. Compared with adults and adolescents, the clinical signs and course of disease in children exhibit differences and occasionally untypical symptoms. Therefore, JMG is often missed and the diagnosis delayed. Isolated ocular symptoms are frequent at onset, spontaneous remission or intermittent symptoms over the longer period of time can occur. Very young children may present with generalized muscle weakness already during the second year of life and in this patient group, specific antibodies can only be slightly increased or even negative. Existing therapeutic options include immunosuppressive therapy and thymectomy but potential long-term side effects on the growing organism and possible influence on immune response in very young children should be considered. Specific clinical symptoms, diagnostic procedures, and a therapeutic approach with consideration of this age group's specificities are discussed.
\end{abstract}

\section{Introduction}

The first description of myasthenia gravis (MG) with onset in childhood originates from Erb in $1879 .^{1}$ The main action of disease is production of antibodies ( $\mathrm{Ab}$ ) against the components of postsynaptic membrane, predominantly the binding acetylcholine receptor (AChR). As a consequence, abnormal muscular endurance and exercise intolerance are the main symptoms of disease. Children may present with isolated symptoms such as ocular problems (ptosis, ophthalmoplegia) or generalized muscle weakness, aggravating in the course of the day or during/after exercise. According to the classification, in adults, MG is divided into five groups ${ }^{2}$ : ocular myasthenia (grade I), mild (grade IIa), moderate (grade IIb), severe weakness other than ocular (grade III), and very severe

received

October 4, 2013

accepted after revision

November 14, 2013

published online

January 27, 2014

weakness defined by intubation with or without mechanical ventilation (grade IV). The definition of juvenile myasthenia gravis (JMG) includes infants, children, and adolescents aged 0 to 19 years. ${ }^{3,4}$ JMG patients are subdivided according to the occurrence of the first symptoms as prepubertal (first symptoms before the age of 12 years) and postpubertal (first symptoms after the age of 12 years ). Although spontaneous remission in prepubertal patients, presenting only by ocular symptoms, is frequent, the early diagnosis is important to start an appropriate therapy in order to improve the clinical course and to prevent generalization. In contrast to adult MG, very young children with JMG presenting generalized symptoms may have no or only slight elevated titer of specific antibodies and particularly in this patient group, the diagnosis is often delayed or the patients are first diagnosed as

(c) 2014 Georg Thieme Verlag KG Stuttgart · New York
DOI http://dx.doi.org/ 10.1055/s-0033-1364181. ISSN $0174-304 X$. 
having myopathy or another inflammatory disease such as Guillain-Barré syndrome or brain stem encephalitis. ${ }^{5}$ Patients with negative specific antibodies and prominent bulbar or facial symptoms may mislead to the diagnosis of a congenital myasthenic syndrome (CMS).

Recently, several reviews have been published, ${ }^{3,4,6,7}$ pointing out the distinct features of JMG. In this overview, we try to present specific clinical symptoms that should help pediatricians and neuropediatricians to provide an early diagnosis. We also propose recommendations for standard diagnostic workup, treatment strategies, and possible differential diagnosis in case of JMG.

\section{Epidemiology}

Recent review showed an incidence of MG for the population in Europe to be around 30/1,000,000/year, the incidence in children and adolescents aged 0 to 19 (JMG) being between 1.0 and 5.0/1,000,000/year. ${ }^{8}$ Only few studies reported the incidence of MG outside Europe, ranging from 3 to 9.1/ 1,000,000/year (countries included: Tanzania, Hong Kong, Japan, North America, Cuba, Curacao, and Aruba). ${ }^{8}$

There is a correlation between ethnical origin and presentation and prevalence of the disease: the occurrence of the first symptoms varied in European/Asian/North American population. In Asian population, up to $50 \%$ of $\mathrm{MG}$ patients present with the first symptoms in childhood (age peak between 5 and 10 years); no difference in sex distribution has been observed. In this population, more than $70 \%$ of cases are restricted to ocular symptoms and benign course is common., 3,10 Also among Caucasian prepubertal patients, presenting only with ocular symptoms, spontaneous remission is frequent and ranges from 15 to $34.7 \%{ }^{6}$ In prepubertal children, similar to elderly patients ( 65 or 70 years or older), no sex predominance has been found. In postpubertal children, female predominance, as also in many others autoimmune disorders, is present. $^{3,9}$

The JMG population among African American shows differences compared to the Asian and European population. African American children demonstrated poorer response to thymectomy than Caucasian children and lower remission rates. ${ }^{11}$ In this population, female predominance is present in all age groups. ${ }^{11}$ In the past studies, spontaneous remission included both, patient receiving no treatment and remission after pharmacotherapy. ${ }^{6}$

In postpubertal children, the clinical course is similar to adult-onset MG. The patients frequently present with ocular symptoms at the onset; generalized-muscle weakness develops in up to $80 \%$ in the course of the disease. ${ }^{3-6,12}$ Postpubertal patients also have an increased association with other autoimmune disorders such as autoimmune thyroid disease, juvenile dermatomyositis, or rheumatoid arthritis. ${ }^{13-15}$ Recently, the association between MG and neuromyelitis optica has also been reported. ${ }^{16,17}$

Different clinical presentation related to ethnic origin may be generated by specific human leukocyte antigen: DRw9 and DRw13 in Japanese, Bw46DR9 in Chinese patients, DQ8 and
DR3 in affected Caucasian, and DR5 in African American children. $^{18-21}$

\section{Clinical Presentation}

A typical clinical symptom of abnormal neuromuscular transmission is a fatigability that occurs acute or subacute. The first symptoms may develop as early as in the first year of life. ${ }^{6}$ Prior to the appearance of symptoms, children have normal psychomotor development, sometimes febrile or afebrile infection might be antedate. Clinical presentation depends on the muscle group affected: ocular problems such as ptosis or ophthalmoplegia, bulbar muscle weakness, respiratory muscle involvement (respiratory insufficiency), and proximal symmetrical muscle weakness.

Ocular symptoms are common at onset. The definition of ocular MG is restricted to the ocular symptoms for 2 years without becoming generalized ${ }^{22}$ and therefore, the differentiation between ocular or generalized myasthenia is not possible at the time first symptoms occur. Our personal observation is that some children develop generalized symptoms after the period of 3 years; therefore, longer follow-up is recommended. Here, the patients may present with isolated, fluctuating unilateral or bilateral ptosis (-Fig. 2A and C), associated ocular symptoms such as ophthalmoplegia, strabismus or lid twitch may be present. Ptosis usually aggravates after physical activity. Children may complain about double vision, particularly during prolonged reading or in the course of the day. Sometimes, children may present to the pediatrician by having difficulties (or being insecure) in climbing stairs because of diplopia. Ptosis induces children to recline their heads so they can see much better. About $50 \%$ of children with ocular symptoms develop systemic or bulbar muscle involvement within 2 years after onset. ${ }^{13,14,23}$

Children with bulbar muscle involvement try to avoid food that is difficult to chew or swallow (meat, apples), liquids may regurgitate through the nose. In case of frequent coughing during meal, the risk of aspiration must be considered. Speech becomes slurred, quiet, and nasal after prolonged talking or in the evening. Because of facial muscle weakness, children may appear unhappy and have difficulties to smile appropriately. In case of generalized muscle weakness, patients have problems to walk a normal distance or to run, have difficulties in climbing stairs or to rise from a squatting position. If upper extremities are affected, problems in personal hygiene may be present: children have difficulties to brush their teeth or to wash and comb their hair. In the physical examination, children may appear normal if examined after rest; repetitive assessment of muscle strength with standardized tests is recommended (Besinger score ${ }^{24}$ ) but not easy to perform in very young children. Rarely, patients may complain of generalized fatigue as a major ailment, muscle pain is uncommon. ${ }^{25}$

There is worsening of symptoms in the course of the day or prolonged use of the affected muscles and improvement after rest ( $\mathbf{- T a b l e ~} \mathbf{1}$ )

Many factors may aggravate myasthenic symptoms: systemic illness, pregnancy, the menstrual cycle, drugs that affect neuromuscular transmission (may be downloaded under 
Table 1 Clinical symptoms suggestive for JMG

\begin{tabular}{|l|l|}
\hline Prepubertal children & $\begin{array}{l}\text { Alternating ocular symptoms are frequent } \\
\rightarrow \text { ptosis, double vision, blurred vision } \\
\text { Generalized muscle weakness is rare }\end{array}$ \\
\hline At every age & $\begin{array}{l}\text { Unilateral or bilateral ptosis ptosis, ophthalmoplegia facial weakness, } \\
\text { hypomimia bulbar symptoms, exercise intolerance, respiratory problems, } \\
\text { cough insufficiency, respiratory insufficiency possible diurnal alternation } \\
\text { of symptoms (worsening in the evening, improvement after rest), } \\
\text { worsening of symptoms during infections/fever/exercise/increased temperature }\end{array}$ \\
\hline
\end{tabular}

Abbreviation: JMG, juvenile myasthenia gravis.

www.dmg.org or www.mga-charity.org), increased temperature, hyperthyroidism or hypothyroidism, and emotional upset. ${ }^{25}$ Some of the agents that should be avoided in JMG are: Dpenicillamine, aminoglycosides, ciprofloxacin, tetracycline, erythromycin, clindamycin, interferon alpha, $\beta$-blockers (propranolol, atenolol, and timolol eyedrops), neuromuscular blocking agents (atracurium, vecuronium), antiarrhythmic drugs (verapamil, quinidine, and procainamid), magnesium, chloroquine, statins, phenytoin, and lithium.

Where there is a rapid increase in bulbar and respiratory muscle involvement, risk of so called "myasthenic crisis" is present. In this situation, intubation or ventilatory support may be required. In addition to respiratory insufficiency and generalized muscle weakness, the patients usually suffer from tachycardia, obstipation, and ptosis. The rapid deterioration of weakness is associated with viral or bacterial infections, physical stress, or changes in medication (initiation or discontinuation of steroids, change of acetylcholinesterase $[\mathrm{AChE}]$ dose) or by using drugs that affect neuromuscular transmission, as already listed. In approximately 30 to $40 \%$ of MG cases, no trigger can be identified. ${ }^{25}$

"Cholinergic crisis" is a consequence of overdosing in AChE medication. Here, increased bronchial secretion, muscular fasciculation, warm and overheated skin, and opposite to myasthenic crises, bradycardia and diarrhea are present. It is often difficult to distinguish between myasthenic crisis and cholinergic crisis, here the administration of edrophonium is possible, but only in an intubated and ventilated patient. ${ }^{25}$ Both "myasthenic" and "cholinergic" crises require intensive care of the patients.

\section{How to Diagnose Juvenile Myasthenia Gravis?}

To diagnose JMG, the combination of a thorough history, repeated physical examination, and neurophysiological investigations (repetitive nerve stimulation tests), as well as antibody samples are helpful clues. Peripubertal and postpubertal children may present with very suggestive clinical symptoms and have in up to $90 \%$ positive AChR antibodies in case of generalized disease., ${ }^{4,25}$ The diagnosis in younger children constitutes a particular challenge as nonspecific symptoms may be present or the antibodies are only minimal elevated or even normal, also in case of severe generalized muscle weakness. In this population, the clinical symptoms may be very similar to genetic determinate CMS and therefore, not always easy to differentiate. Recently, we gave an overview concerning this heterogeneous group of patients and specific clinical clues that should help to provide the right diagnosis. $^{26}$

\section{Diagnostic Workup}

\section{Serology}

In case of positive antibodies against AChR, MuSK (musclespecific kinase), and Titin, the diagnosis is almost certain. In cases of predominant proximal weakness in lower extremites with areflexia, also voltage-gated calcium-channel antibodies should be detected. Rarely, false-positive tests have been reported in patients with autoimmune liver disease, systemic lupus, inflammatory neuropathies, amyotrophic lateral sclerosis, rheumatoid arthritis receiving penicillamine, patients with thymoma without MG. ${ }^{25}$ In one patient with positive $\mathrm{AChR}-\mathrm{Ab}$ and delayed motor development with bulbar symptoms, we diagnosed a brain tumor (brain stem glioma). In adult seronegative patients, low-sensitivity AChR-Ab and low-density lipoprotein receptor-related protein 4 (Lrp4) has recently been reported. The detection of these antibodies is available only in specialized laboratories and should be initiated in seronegative JMG patients with distinct clinical symptoms and abnormal neurophysiological findings or vice versa. ${ }^{27-29}$ To our knowledge, to date, only one JMG patient positive for Lrp4-Ab has been reported (17 years of age). ${ }^{29}$ Here, larger number of patients and long-term follow-up are necessary to clarify the clinical and therapeutic implications of low-sensitivity AChR-Ab and Lrp4-Ab in pediatric population.

In JMG patients with seronegative MG, most often young children with an ocular myasthenia, antibodies are not helpful to differentiate from CMS because they may be negative in up to $50 \%{ }^{11,13,23,30,31}$ Titin antibodies are frequent negative because a thymoma (associated with Titin antibodies in adulthood) is extremely rare at this young age.,23,25 The transient neonatal AChR-Ab can be detected in case of maternal myasthenia; the careful history and examination of the mother is helpful. The symptoms in neonates usually develop during the first 3 days after delivery, last usually 2 to 4 weeks, but may last for up to 3 months. ${ }^{3,25}$

MuSK antibodies are also rare in children and appear to be associated with a more severe disease, affecting mostly facial and bulbar weakness as well as frequent respiratory crises in 
association with generalized muscle weakness. ${ }^{3,4,32,33}$ Here, females are predominantly affected and most pediatric cases have been reported within adult series. ${ }^{34-37}$ Seroconversion over time has been described in cases of children who developed MuSK antibodies after thymectomy for AChRseropositive $\mathrm{MG}^{38}$

\section{Electrophysiology}

To demonstrate abnormal neuromuscular transmission, the next step is to perform a repetitive stimulation test (RNS), usually with low frequency of $3 \mathrm{~Hz}$. We investigate distal nerves (N. medianus, N. ulnaris) and/or proximal nerves ( $N$. accessorius); the extension of these investigations depends on the age and compliance of the patient. In case of a pathological decrement response (decline from the first to the fifth amplitude more than $10 \%$ ), the result is very suggestive of a transmission defect. To differentiate between CMS and autoimmune myasthenia, the additional clinical history and antibody findings are used to guide the diagnosis.

Single fiber electromyography (SFEMG) is more sensitive than RNSs and can provide pathological results in patients with normal decrement response in RNSs. SFEMG is technically more difficult in children and could be only performed in sedated children or in postpubertal patients with very good compliance; therefore, we need strong indications before performing these neurophysiological investigations. ${ }^{26}$ Normal jitter in SFEMG in a weak (affected) muscle excludes $\mathrm{MG}^{25}$

\section{Pharmacological Testing}

Intravenous (IV) application of edrophonium (also known as Tensilon test) inhibits the action of AChE, allowing acetylcholine to have a more prolonged contact with AChR on the postsynaptic muscle membrane. It is indicated from the $1 \mathrm{st}$ year of life and, because of possible side effects (increased salivation, hypotension, sweating, nausea, and bradycardia), has to be performed under intensive care conditions within resuscitation educated stuff. Children should have obvious clinical symptoms (ptosis, bulbar symptoms), otherwise a correct interpretation is not possible. ${ }^{26}$ Improvement after edrophonium can be also seen in CMS, the Lambert-Eaton myasthenic syndrome, motor neuron disease, intracranial aneurysm, brain stem lesions, cavernous sinus tumors, endstage renal disease, and in muscle diseases affecting the ocular muscle. ${ }^{25}$

The optimal dose of edrophonium varies among patients and cannot be predetermined. Recommended dose is 0.15 $\mathrm{mg} / \mathrm{kg}$ subcutaneous in newborns and IV in infants, preceded by an intravenous test dose of $0.01 \mathrm{mg} / \mathrm{kg}$ and then depending on effects increasing with $0.15 \mathrm{mg}$ steps to a maximum of $0.6 \mathrm{mg}$. In children older than 1 year and with bodyweight below $34 \mathrm{~kg}$ the dose is 0.5 to $1.0 \mathrm{mg}$ IV initially, then increasing by $1 \mathrm{mg}$ to a maximum of $5 \mathrm{mg}$. In adolescents/ adults with bodyweight above $34 \mathrm{~kg}$ the dose is $2.0 \mathrm{mg}$ IV initially, then increasing by 2 to $4 \mathrm{mg}$ to a maximum of 10 mg. $6,25,26$
Because of possible side effects, we do not perform IV or intramuscular edrophonium-testing in very young children. Here, we start pyridostigmine as an oral, age-adapted dose, and increase it gradually over the period of several days. This is a good alternative in very young children, 1 hour after intake of medication children are monitored for their vital signs. In case of positive effect, there is an improvement of muscular weakness within 30 to 60 minutes. This regime may be also a good alternative if edrophonium is not available, as the case in Australia. ${ }^{39}$

\section{Chest Imaging}

Before the steroid therapy, both subcutaneous testing and chest X-ray should be performed to exclude fluent tuberculosis. Computed tomography (CT) or magnetic resonance imaging (MRI) of the chest to check for thymus hyperplasia or thymoma are needed. Because of radiation exposure, we prefer thymus MRI in children.

\section{Differential Diagnosis of Juvenile Myasthenia Gravis}

In patients with fluctuating ptosis, particularly if this alternates between the eyes, the diagnosis is relatively clear. In case of additional ocular or bulbar symptoms or systemic muscle weakness, possible differential diagnoses are listed in -Table 2.

\section{Therapeutic Management}

Until now, therapeutic standards for treatment of JMG have been adopted from adult patients. ${ }^{7,40} \mathrm{JMG}$, particularly the group of prepubertal children, shows to some extent different presentation and the course of the disease. Therapeutic considerations and decisions must take into account the distinctiveness of this population, in particular concerning growth and immune-system development. The treatment regimen depends on clinical presentation: in case of ocular symptoms only (fluctuating ptosis, ophthalmoplegia), the therapy consists of AChE inhibitors, less frequently steroids. Some patients profit from intermittent steroid therapy only (personal observation, case 2). If generalized muscle weakness is present, multimodal therapy includes AChE inhibitors, immunosuppressive and immunomodulating agents as well as surgical treatment (-Table $\mathbf{3}$ ).

\section{Acetylcholinesterase Inhibitors}

Pyridostigmine is the first-line therapy in JMG for long time use and is administered orally. AChE inhibitors lead to a prolonged activity of acetylcholine (ACh) in the synaptic cleft by blocking hydrolysis of ACh. Initial dosage is 0.5 to $1 \mathrm{mg} / \mathrm{kg} / \mathrm{d}$ every 4 to 6 hours, a daily increase up to 5 to $7 \mathrm{mg} / \mathrm{kg} / \mathrm{d}$ is possible, maximal $300 \mathrm{mg} / \mathrm{d}^{6,40}$ In older children/adults, the maximum starting dose is $60 \mathrm{mg} 3$ to 4 times daily and absolute maximum recommended dose in adults is $120 \mathrm{mg}$ every 3 hours during the daytime. ${ }^{7}$ Possible side effects are: nausea, sweating, papillary constriction, diarrhea, hypersalivation, hypotension, and bradycardia. In case, symptoms are already present in the morning or during night sleep, a long-acting preparation is available but we side frequent cholinergic side effects in 
Table 2 Possible differential diagnosis for JMG

\begin{tabular}{|c|c|c|}
\hline Differential diagnosis & Clinical clues & Diagnostic workup \\
\hline CMS & $\begin{array}{l}\text { Family history } \\
\text { Ethnicity } \\
\text { Occurrence of the first symptoms } \\
\text { Additional findings: scoliosis, joint } \\
\text { contractures, apneas during febrile } \\
\text { infections }\end{array}$ & Genetic testing for CMS \\
\hline Congenital myopathy & & $\begin{array}{l}\text { CK, muscle biopsy } \\
\text { (discuss also if CK normal) }\end{array}$ \\
\hline Mitochondrial myopathy & $\begin{array}{l}\text { Metabolic workup, further organ } \\
\text { manifestation? }\end{array}$ & Muscular biopsy \\
\hline Myotonic dystrophy & $\begin{array}{l}\text { Myotonic discharges in EMG? } \\
\text { Relatives affected? }\end{array}$ & $\begin{array}{l}\text { Myotonic discharges in EMG? } \\
\text { During the daytime Genetic testing }\end{array}$ \\
\hline Lambert-Eaton myasthenic syndrome & $\begin{array}{l}\text { Abnormal muscle stretch reflexes } \\
\text { Autonomic symptoms (dry mouth) }\end{array}$ & $\begin{array}{l}\text { Search for neoplasm } \\
\text { Ca-channel antibodies (VGCC) }\end{array}$ \\
\hline $\begin{array}{l}\text { Chronic progressive bulbar paralysis } \\
\text { (Fazio-Londe disease) }\end{array}$ & Involvement of additional cranial nerves? & Genetic testing \\
\hline Botulism & Vegetative symptoms? & Toxin verification \\
\hline Guillain-Barré syndrome & $\begin{array}{l}\text { No improvement of weakness after rest } \\
\text { Diminished or absent muscle stretch reflexes }\end{array}$ & $\begin{array}{l}\text { Cerebrospinal fluid } \\
\text { Nerve conduction velocity }\end{array}$ \\
\hline Brainstem tumor & Associated neurological symptoms & $\begin{array}{l}\text { Cranial magnetic resonance } \\
\text { imaging }\end{array}$ \\
\hline
\end{tabular}

Abbreviations: CK, creatine kinase; CMS, congenital myasthenic syndrome; EMG, electromyography; JMG, juvenile myasthenia gravis; VGCC, voltagegated calcium-channel.

a Roma founder mutation (c.1267delG) in the epsilon subunit gene CHRNE.

${ }^{\mathrm{b}}$ To date, in only approximately $50 \%$ of patients with CMS known mutations can be found. ${ }^{26}$

children under $50 \mathrm{~kg}$ weight. In case of hypotension and bradycardia atropine may be given.

\section{Immunomodulation/Immunosuppression}

Prednisone and prednisolone are the first-line therapy in children with persisting symptoms. The starting dosage is 0.5 to $1 \mathrm{mg} / \mathrm{kg}$ (maximum $30 \mathrm{mg} / \mathrm{d}$ ), with a possible increase up to $2 \mathrm{mg} / \mathrm{kg} / \mathrm{d}$ (maximum $60-80 \mathrm{mg} / \mathrm{d}$ ). To minimize side effects, we recommend administering steroids (particularly in peri/postpubertal patients or if obesity is present) every second day. The patients are at risk of worsening during the first weeks of steroid therapy and in case of severe weakness or respiratory or bulbar involvement the patients should remain in inpatient care during the first 2 week after steroid is initiated. Because of significant side effects in case of chronic use, such as growth retardation, weight gain, hypertonia, cataracts, hyperinsulinaemia, steroid therapy should be kept short term.

The role of IV methylprednisolone pulses has not been well studied in children or in adults with myasthenia and therefore cannot be recommended. ${ }^{41}$ In the past studies, azathioprine was the most commonly used cytotoxic drug in JMG. ${ }^{10,13,23,31,39,42,43}$ Recommended initial dose is 0.5 to $1 \mathrm{mg} / \mathrm{d}$; increase of $0.5 \mathrm{mg} / \mathrm{kg} / \mathrm{d}$ every 4 weeks up to $2.5 \mathrm{mg} / \mathrm{kg} / \mathrm{d}$ (maximum 150-200 mg/d) in two divided doses is possible. The onset of therapeutic response is often delayed by 4 to

Table 3 Therapeutic strategies for JMG

\begin{tabular}{|l|l|l|l|}
\hline Clinical presentation & First-line therapy & Additional therapy & Comment \\
\hline Isolated ocular symptoms & Pyridostigmine & $\begin{array}{l}\text { Steroids, try intermittent } \\
\text { administration }\end{array}$ & $\begin{array}{l}\text { Consider thymectomy } \\
\text { if long-term immunosuppression } \\
\text { is needed or thymus-pathology } \\
\text { is present }\end{array}$ \\
\hline Generalized muscular weakness & $\begin{array}{l}\text { Pyridostigmine } \\
\text { + Steroids }\end{array}$ & $\begin{array}{l}\text { Start long-term } \\
\text { immunosuppression }\end{array}$ & Prepare thymectomy \\
\hline $\begin{array}{l}\text { Moderate-to-severe bulbar symptoms } \\
\text { or respiratory insufficiency }\end{array}$ & $\begin{array}{l}\text { Pyridostigmine } \\
\text { + Steroids } \\
+ \text { Plasmapheresis }\end{array}$ & $\begin{array}{l}\text { Start long-term } \\
\text { immunosuppression }\end{array}$ & Prepare thymectomy \\
\hline
\end{tabular}

Abbreviation: JMG, juvenile myasthenia gravis. 
12 months. ${ }^{7}$ Possible side effects are flu-like symptoms, gastrointestinal disturbances, pancreatitis, elevation of liver enzymes, and hair loss. Leucopenia, anemia, and thrombocytopenia usually respond to drug reduction or withdrawal. Patients with mutations of the thiopurine methyltransferase gene are at risk of azathioprine-induced bone marrow suppression, and therefore, genetic testing could be preformed before the therapy. During the therapy, leukocyte counts and liver function tests need to be monitored regularly. Because of reported malignancy associated with chronic azathioprine use (fatal T-cell lymphoma), we do not recommend that the use be prolonged beyond the period of 1 to 2 years. ${ }^{40}$

In case of azathioprine, intolerance or unresponsiveness, other immunosuppressive agents are in use as a second-line therapy. Cyclosporine A is considered as a second-line drug after azathioprine, ${ }^{7}$ but there have been only a few pediatric case series in JMG. ${ }^{23,44}$ Cyclophosphamide was used only occasionally in JMG, side effects are frequent and severe (hair loss, nausea, vomiting, myelosuppression, cystitis, and malignancy) and is, as well in adults, restricted to refractory cases. ${ }^{7}$ Mycophenolate mofetil (MMF) and tacrolimus have also exhibited benefits in more recently published studies and because of a milder side effect profile are deemed a possible option. ${ }^{39,45-47}$ In refractory, JMG rituximab has been used. ${ }^{48,49}$

Because of milder side effects profile and shorter latency of action, newer immunosuppressants have to be tested in JMG over a longer period of time and may be a good alternative in children with side effects under azathioprine. Recent reports including children with JMG show tendency for use of MMF as steroid sparing agent. ${ }^{47,50}$

Intravenous immunoglobulin (IVIg) and plasma exchange (PEX) are used as a short-term therapy in moderate-to-severe MG or during myasthenic crises. PEX usually induces improvement within days and can be repeated in patients who fail to respond to other therapies. ${ }^{5}$ Usually, a total of five PEX every 2nd day are recommended, we administrate 8 to 10 single-volume exchanges over a period of 14 days. PEX is restricted to pediatric centers with multidisciplinary treatment facilities.

The standard dose of IVIg has been $2 \mathrm{~g} / \mathrm{kg}$ administrated over the period of 2 to 5 days, maximum dose $150 \mathrm{~g}$. This regiment can be repeated every 4 to 8 weeks in patients who have failed to respond to other therapies.

\section{Surgical Treatment}

Thymus plays a role in the pathophysiology of MG, in JMG thymic hyperplasia is evident in $83 \%$ of patients, in $3.8 \%$ thymomas could be detected. ${ }^{23}$ Thymectomy is followed by improvement in most cases: remission rates were higher in children after thymectomy than the rate of spontaneous remission. 11,14,39,50,51 Thymectomy performed within the first year after onset is associated with higher remission rates. $^{50,52-54}$ Therefore, thymectomy is recommended as early as possible in case of generalized weakness. Before thymectomy patients should be clinically stable, otherwise postoperative complications up to myasthenic crises are possible. Long-term follow-up studies in prepubertal children after thymectomy are still pending. Here, the question of possible benefit and long-term side effects of immunosuppressive treatment should be discussed. Although in prepubertal patients, the spontaneous remission rate without surgical therapy is higher, the right time for thymectomy should not be missed.

After thymectomy in infancy because of open heart surgery, children showed a delayed immune response to new antigens such as tick-bone encephalitis-vaccine. ${ }^{55}$ At time, no studies concerning influence of thymectomy on immune response to vaccination particularly in very young children with JMG are present. The question of immunological side effects after thymectomy during early childhood is still to be discussed. ${ }^{3}$ In ocular myasthenia, thymectomy can be an option if the patients are resistant to medical treatment ${ }^{7,45,56}$ or side effects of immunosuppressive therapy cannot be tolerated.

In case of MuSK antibody-positive MG, no clear benefit of thymectomy has been proven, ${ }^{7,34}$ here, further deterioration of symptoms after thymectomy was reported. ${ }^{38}$

Depending on the surgeon's expertise, thoracoscopic techniques or extended transsternal approach are used. We recommend thoracoscopic techniques but these are restricted to specialized centers. Where improvement or remission after thymectomy is lacking, repeated operative exploration of the thymus is necessary. ${ }^{7}$

Children with moderate-to-severe disease need intensive physiotherapy as a supportive therapy after clinical symptoms stabilized. However, in patients with late or inappropriate treatment, constant muscle weakness, swallowing difficulties or exercise intolerance over years can be present (personal observation) (-Table 3 ).

\section{Case Reports}

\section{Case 1: Prepubertal Patient with Generalized Weakness}

The girl was born at term after an uneventful pregnancy. At the age of 18 months, bilateral ptosis and ophthalmoplegia developed, following a febrile infection. Initially, she was treated with pyridostigmine and ocular symptoms improved. Because of negative antibody testing for myasthenia, CMS as a possible diagnosis was first considered. After another febrile infection, the patient developed additional bulbar symptoms: hypersalivation, chewing difficulties, and facial hypomimia ( - Fig. 1A). Symptoms worsened during the course of the day or after prolonged physical activity, no clear improvement of the symptoms was observed after rest. Although pyridostigmine dosage was increased, the symptoms proceeded and she developed muscarinic side effects (diarrhea, hypersalivation). Repeated analysis for AChR-Ab was now tested slight positive $(0.6 \mathrm{mmol} / \mathrm{L}$, normal range $<0.4 \mathrm{mmol} / \mathrm{L})$. Steroid treatment and azathioprine were added to pyridostigmine and after the period of 3 weeks her symptoms clearly improved. On followup, at the age of 2 years she was symptom free (-Fig. 1B). Steroid therapy was tapered during following 4 months. At the age of 3 years, her condition worsened again after a febrile infection under therapy with azathioprine and 

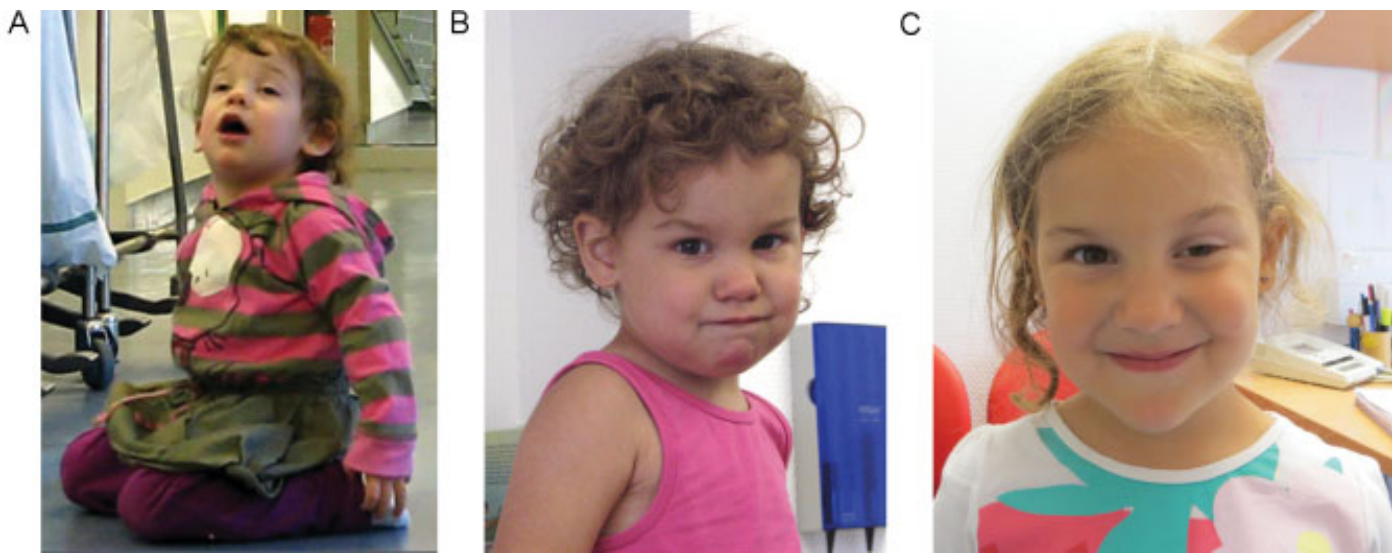

Fig. 1 (A) Case 1, 18-month-old with generalized muscle weakness, bilateral ptosis, and ophthalmoplegia. (B) She improved under therapy with pyridostigmine, prednisone, and azathioprine 4 months later. (C) At follow-up at the age of 4 years, 1 month after thymectomy, left-sided ptosis was the only present symptom.
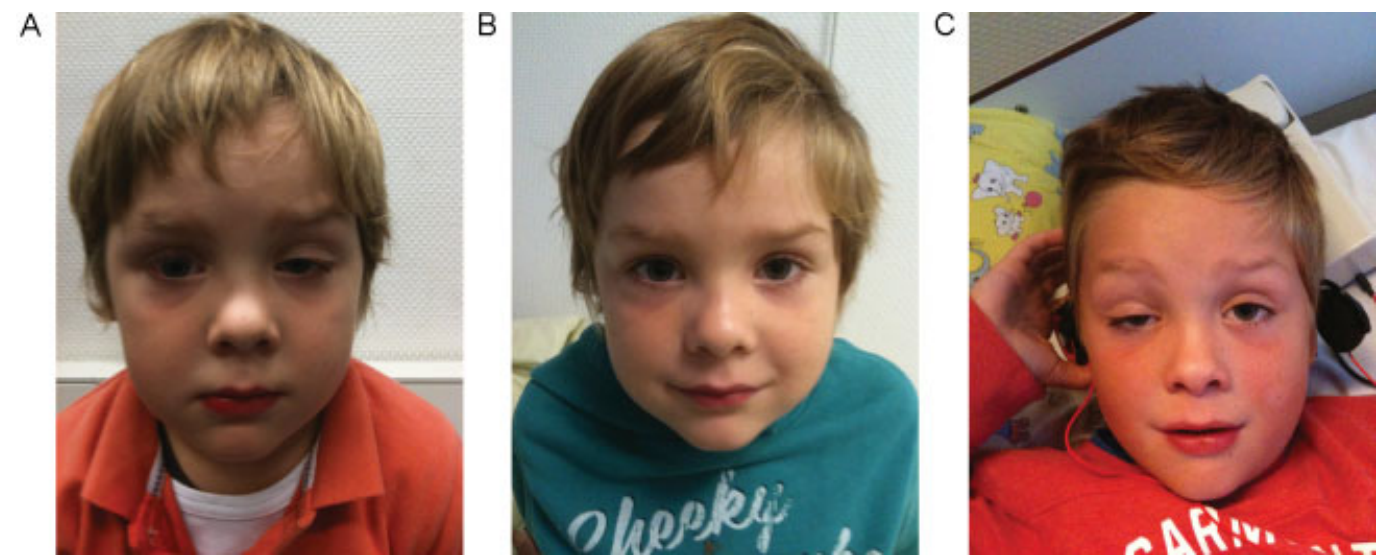

Fig. 2 (A) A boy developed at the age of 5 years one-sided ptosis and (B) first improved under monotherapy with pyridostigmine. (C) Two-months later, he developed bilateral ptosis and axial muscle weakness. Improvement under therapy with prednisone; pyridostigmine showed no effect.

pyridostigmine. She improved once again after reinitiating of prednisone. Meanwhile, she has reached the age of 4 years and we indicate thymectomy because of moderate muscle weakness and bulbar symptoms. At the first visit 1 month after thymectomy at our outpatient clinic, she had normal muscular endurance, one-sided ptosis was present as the only residual symptom (- Fig. $\mathbf{1})$.

\section{Case 2: Prepubertal Patient with Fluctuating Ptosis}

At the age of 23 months, a boy developed intermittent unilateral ptosis. Initial pyridostigmine therapy yielded no clear improvement in the symptoms, he showed benefit from prednisone therapy over the period of 4 weeks. AChR-Ab were first negative, became positive over the time (range, 0.5-0.62 $\mathrm{mmol} / \mathrm{L}$ ), normalized thereafter. Between the age of 2 and 4 years, repeated ptosis improved under intermittent prednisone therapy. At the age of 5 years, he developed ptosis immediately after intermittent prednisone therapy was stopped. This time monotherapy with pyridostigmine (1.5 $\mathrm{mg} / \mathrm{kg}$ ) first induced full remission (-Fig. 2A and 2B). Two months after remission, bilateral ptosis and axial muscle weakness occurred (-Fig. $\mathbf{2 C}$ ) and prednisone therapy was started for a longer period of time. Because of aggravated symptoms and MRI finding of thymus hyperplasia, thymectomy is planned.

\section{Conclusion}

MG in young children and adolescents shows distinct features of clinical signs when compared with adults, and the diagnosis, particularly in very young children can still be a challenge for the pediatrician/neuropediatrician. Because of existing therapeutic options, the early diagnosis is extremely important to start an appropriate therapy so as to prevent further progress of muscle weakness and to achieve full remission.

To some extent, treatment options for adults may be adapted for children, but specific pathophysiology differences must be considered in this population group. In term of achieving a remission, there should be a balance in the use of immunosuppressive and immunomodulating therapy because of their possible long-term side effects on the growing organism. 
Evidence-based data for children and adolescents with JMG are still pending and management should urgently be made by taking into account specificities of this age group. Furthermore, long-term follow-up studies are necessary to improve our knowledge in this rare disorder.

\section{References}

1 Erb W. Zur Kasuistik der bulbaeren Lähmungen. Ueber einen neuen, wahrscheinlich bulbaeren Symptomenkomplex. Arch Psychiatr Nervenkr 1879;9:336-350

2 Osserman KE, Genkins G. Studies in myasthenia gravis: review of a twenty-year experience in over 1200 patients. Mt Sinai J Med 1971;38(6):497-537

3 Evoli A. Acquired myasthenia gravis in childhood. Curr Opin Neurol 2010;23(5):536-540

4 Finnis MF, Jayawant S. Juvenile myasthenia gravis: a paediatric perspective. Autoimmune Dis 2011;2011:404101

5 Andrews PI. Autoimmune myasthenia gravis in childhood. Semin Neurol 2004;24(1):101-110

6 Chiang LM, Darras BT, Kang PB. Juvenile myasthenia gravis. Muscle Nerve 2009;39(4):423-431

7 Ionita CM, Acsadi G. Management of juvenile myasthenia gravis. Pediatr Neurol 2013;48(2):95-104

8 McGrogan A, Sneddon S, de Vries CS. The incidence of myasthenia gravis: a systematic literature review. Neuroepidemiology 2010; 34(3):171-183

9 Chiu HC, Vincent A, Newsom-Davis J, Hsieh KH, Hung T. Myasthenia gravis: population differences in disease expression and acetylcholine receptor antibody titers between Chinese and Caucasians. Neurology 1987;37(12):1854-1857

10 Zhang X, Yang M, Xu J, et al. Clinical and serological study of myasthenia gravis in HuBei Province, China. J Neurol Neurosurg Psychiatry 2007;78(4):386-390

11 Andrews PI, Massey JM, Howard JF Jr, Sanders DB. Race, sex, and puberty influence onset, severity, and outcome in juvenile myasthenia gravis. Neurology 1994;44(7):1208-1214

12 Kalb B, Matell G, Pirskanen R, Lambe M. Epidemiology of myasthenia gravis: a population-based study in Stockholm, Sweden. Neuroepidemiology 2002;21(5):221-225

13 Lindner A, Schalke B, Toyka KV. Outcome in juvenile-onset myasthenia gravis: a retrospective study with long-term follow-up of 79 patients. J Neurol 1997;244(8):515-520

14 Rodriguez M, Gomez MR, Howard FM Jr, Taylor WF. Myasthenia gravis in children: long-term follow-up. Ann Neurol 1983;13(5): 504-510

15 Christensen PB, Jensen TS, Tsiropoulos I, et al. Associated autoimmune diseases in myasthenia gravis. A population-based study. Acta Neurol Scand 1995;91(3):192-195

16 Jarius S, Wildemann B. AQP4 antibodies in neuromyelitis optica: diagnostic and pathogenetic relevance. Nat Rev Neurol 2010;6(7): 383-392

17 Leite MI, Coutinho E, Lana-Peixoto M, et al. Myasthenia gravis and neuromyelitis optica spectrum disorder: a multicenter study of 16 patients. Neurology 2012;78(20):1601-1607

18 Shinomiya N, Nomura Y, Segawa M. A variant of childhood-onset myasthenia gravis: HLA typing and clinical characteristics in Japan. Clin Immunol 2004;110(2):154-158

19 Chen WH, Chiu HC, Hseih RP. Association of HLA-Bw46DR9 combination with juvenile myasthenia gravis in Chinese. J Neurol Neurosurg Psychiatry 1993;56(4):382-385

20 Wong V, Hawkins BR, Yu YL. Myasthenia gravis in Hong Kong Chinese. 2. Paediatric disease. Acta Neurol Scand 1992;86(1):68-72

21 Christiansen FT, Pollack MS, Garlepp MJ, Dawkins RL. Myasthenia gravis and HLA antigens in American blacks and other races. J Neuroimmunol 1984;7(2-3):121-129
22 Luchanok U, Kaminski HJ. Ocular myasthenia: diagnostic and treatment recommendations and the evidence base. Curr Opin Neurol 2008;21(1):8-15

23 Evoli A, Batocchi AP, Bartoccioni E, Lino MM, Minisci C, Tonali P. Juvenile myasthenia gravis with prepubertal onset. Neuromuscul Disord 1998;8(8):561-567

24 Besinger UA, Toyka KV, Hömberg M, Heininger K, Hohlfeld R, Fateh-Moghadam A. Myasthenia gravis: long-term correlation of binding and bungarotoxin blocking antibodies against acetylcholine receptors with changes in disease severity. Neurology 1983; 33(10):1316-1321

25 Sanders DB, Massey JM. Clinical features of myasthenia gravis. Handb Clin Neurol 2008;91:229-252

26 Schara U, Della Marina A, Abicht A. Congenital myasthenic syndromes: current diagnostic and therapeutic approaches. Neuropediatrics 2012;43(4):184-193

27 Leite MI, Jacob S, Viegas S, et al. IgG1 antibodies to acetylcholine receptors in 'seronegative' myasthenia gravis. Brain 2008;131(Pt 7):1940-1952

28 Higuchi O, Hamuro J, Motomura M, Yamanashi Y. Autoantibodies to low-density lipoprotein receptor-related protein 4 in myasthenia gravis. Ann Neurol 2011;69(2):418-422

29 Pevzner A, Schoser B, Peters K, et al. Anti-LRP4 autoantibodies in AChR- and MuSK-antibody-negative myasthenia gravis. J Neurol 2012;259(3):427-435

30 Snead OC III, Benton JW, Dwyer D, et al. Juvenile myasthenia gravis. Neurology 1980;30(7 Pt 1):732-739

31 Ashraf VV, Taly AB, Veerendrakumar M, Rao S. Myasthenia gravis in children: a longitudinal study. Acta Neurol Scand 2006;114(2):119-123

32 Anlar B, Yilmaz V, Saruhan-Direskeneli G. Long remission in muscle-specific kinase antibody-positive juvenile myasthenia. Pediatr Neurol 2009;40(6):455-456

33 Pasnoor M, Wolfe GI, Nations S, et al. Clinical findings in MuSKantibody positive myasthenia gravis: a U.S. experience. Muscle Nerve 2010;41(3):370-374

34 Guptill JT, Sanders DB, Evoli A. Anti-MuSK antibody myasthenia gravis: clinical findings and response to treatment in two large cohorts. Muscle Nerve 2011;44(1):36-40

35 Tsiamalos P, Kordas G, Kokla A, Poulas K, Tzartos SJ. Epidemiological and immunological profile of muscle-specific kinase myasthenia gravis in Greece. Eur J Neurol 2009;16(8):925-930

36 Lavrnic D, Losen M, Vujic A, et al. The features of myasthenia gravis with autoantibodies to MuSK. J Neurol Neurosurg Psychiatry 2005; 76(8):1099-1102

37 Evoli A, Tonali PA, Padua L, et al. Clinical correlates with anti-MuSK antibodies in generalized seronegative myasthenia gravis. Brain 2003;126(Pt 10):2304-2311

38 Kostera-Pruszczyk A, Kwiecinski H. Juvenile seropositive myasthenia gravis with anti-MuSK antibody after thymectomy. J Neurol 2009;256(10):1780-1781

39 Ware TL, Ryan MM, Kornberg AJ. Autoimmune myasthenia gravis, immunotherapy and thymectomy in children. Neuromuscul Disord 2012;22(2):118-121

40 McMillan HJ, Darras BT, Kang PB. Autoimmune neuromuscular disorders in childhood. Curr Treat Options Neurol 2011;13(6): 590-607

41 Lindberg C, Andersen O, Lefvert AK. Treatment of myasthenia gravis with methylprednisolone pulse: a double blind study. Acta Neurol Scand 1998;97(6):370-373

42 Liu W, Tong T, Ji Z, Zhang Z. Long-term prognostic analysis of thymectomized patients with myasthenia gravis. Chin Med J (Engl) 2002;115(2):235-237

43 Mullaney P, Vajsar J, Smith R, Buncic JR. The natural history and opthalmologic involvement in childhood myasthenia gravis at the Hospital for Sick Children. Ophthalmology 2000; 107(3):504-510 
44 Badurska B, Ryniewicz B, Strugalska H. Immunosuppressive treatment for juvenile myasthenia gravis. Eur J Pediatr 1992;151(3): 215-217

45 Pineles SL, Avery RA, Moss HE, et al. Visual and systemic outcomes in pediatric ocular myasthenia gravis. Am J Ophthalmol 2010; 150(4):453-459, e3

46 Hehir MK, Burns TM, Alpers J, Conaway MR, Sawa M, Sanders DB. Mycophenolate mofetil in AChR-antibody-positive myasthenia gravis: outcomes in 102 patients. Muscle Nerve 2010;41(5):593-598

47 Castro D, Derisavifard S, Anderson M, Greene M, Iannaccone S. Juvenile myasthenia gravis: a twenty-year experience. J Clin Neuromuscul Dis 2013;14(3):95-102

48 Wylam ME, Anderson PM, Kuntz NL, Rodriguez V. Successful treatment of refractory myasthenia gravis using rituximab: a pediatric case report. J Pediatr 2003;143(5):674-677

49 Koul R, Al Futaisi A, Abdwani R. Rituximab in severe seronegative juvenile myasthenia gravis: review of the literature. Pediatr Neurol 2012;47(3):209-212
50 Tracy MM, McRae W, Millichap JG. Graded response to thymectomy in children with myasthenia gravis. J Child Neurol 2009; 24(4):454-459

51 Adams C, Theodorescu D, Murphy EG, Shandling B. Thymectomy in juvenile myasthenia gravis. J Child Neurol 1990;5(3): 215-218

52 Andrews PI. A treatment algorithm for autoimmune myasthenia gravis in childhood. Ann N Y Acad Sci 1998;841:789-802

53 Seybold ME. Thymectomy in childhood myasthenia gravis. Ann N Y Acad Sci 1998;841:731-741

54 Hennessey IA, Long AM, Hughes I, Humphrey G. Thymectomy for inducing remission in juvenile myasthenia gravis. Pediatr Surg Int 2011;27(6):591-594

55 Zlamy M, Würzner R, Holzmann $\mathrm{H}$, et al. Antibody dynamics after tick-borne encephalitis and measles-mumps-rubella vaccination in children post early thymectomy. Vaccine 2010;28(51):8053-8060

56 Ortiz S, Borchert M. Long-term outcomes of pediatric ocular myasthenia gravis. Ophthalmology 2008;115(7):1245-1248, e1 